\title{
Alteraciones de la coagulación y marcadores de trombofilia en un paciente con SARS-CoV-2, diabetes tipo 2, hipotiroidismo y flebitis de miembro pélvico izquierdo
}

\author{
Coagulation disorders and thrombophilia markers in a patient with SARS-CoV-2, type 2 \\ diabetes, hypothyroidism and phlebitis of the left pelvic limb
}

\begin{abstract}
Sergio A. Ramírez-García1, Diana García-Cruz², Nory O. Dávalos-Rodriguez², Sabina López-Toledo, Santiago Landeta-Velázquez ${ }^{3}$, José Domínguez-Rodas ${ }^{4}$, Luis J. Flores-Alvarado², Melecio H. Juárez-Pérez y Carlos E. Cabrera-Pivaral ${ }^{1 *}$

${ }^{1}$ Instituto de Nutrición, Universidad de la Sierra Sur, Sistema de Universidades Estatales de Oaxaca (SUNEO), Miahuatlán de Porfirio Díaz, Oaxaca; ${ }^{2}$ Departamento de Biología Molecular y Genómica, Centro Universitario de Ciencias de la Salud (CUCS), Universidad de Guadalajara, Guadalajara, Jalisco; ${ }^{2}$ Laboratorio Estatal de Salud Pública, Servicios de Salud del Estado de Oaxaca, Oaxaca; ${ }^{4}$ Servicios Médicos Profesionales, A.C. Miahuatlán de Porfirio Díaz, Oaxaca; ${ }^{5}$ Departamento de Salud Pública, CUCS, Universidad de Guadalajara, Guadalajara, Jalisco. México
\end{abstract}

Al editor:

Retomamos con gran interés el trabajo de Carrillo-Esper $\mathrm{R}$, et al. ${ }^{1}$ en el cual se hace una revisión de las alteraciones de la coagulación en los pacientes con SARS-CoV-2. En el manejo del paciente con diabetes tipo 2 cobra importancia por la resistencia a la insulina, que conduce a un estado proinflamatorio y protrombótico, potenciando la tormenta de citocinas, así como la inmunotrombosis, a través de una resistencia endocrina múltiple ${ }^{2}$. En este sentido, presentamos el caso de un varón de 55 años, normotenso, con diabetes tipo 2 de 8 años de evolución, originario de la región de la Sierra Sur, Oaxaca, que desarrolló neumonía por SARS-CoV-2 (confirmado por reacción en cadena de la polimerasa y secuenciación; variante $\mathbf{\Delta} 382$ en ORF1a). Presentaba obesidad de grado I e hipotiroidismo por dishormonogénesis, y fue hospitalizado con fenotipo clínico tipo 1 y saturación de oxígeno del $86 \%$. Durante su estancia hospitalaria, se le detectó en el ultrasonograma Doppler de miembro pélvico un trombo superficial en la rama dorsal lateral de la vena safena externa izquierda, con sistema venoso profundo permeable. Se determinaron los biomarcadores para diabetes, inflamación y trombosis (Tabla 1). Su manejo se inició con control de la glucemia con insulina de acción rápida hasta normalizarla en valores de 70-100 mg/dl, y se mantuvo posteriormente con insulina glargina (40 UI por la mañana) y lispro (10 UI por la mañana y por la noche). El hipotiroidismo se controló con levotiroxina (100 mg/24 h). La neumonía se manejó con ivermectina (6 mg/8 h) los primeros 3 días y durante 14 días adicionalmente con dexametasona (8 mg/24 h), azitromicina (500 mg/24 h), hidroxicloroquina (400 mg/12 h de inicio, dos dosis, y posteriormente $200 \mathrm{mg} / 12 \mathrm{~h}$ ) y nitazoxanida $(500 \mathrm{mg} / 12 \mathrm{~h}$ ). Se instaló nefrocardioprotección y tromboprofilaxis con dapagliflozina (10 mg/24 h), ácido acetilsalicílico (100 mg/24 h) y enoxaparina sódica (60 mg/24 h, subcutánea). El paciente se dio de alta a los 18 días con saturación de oxígeno del 96\%, temperatura de $36.5^{\circ} \mathrm{C}$ y ARN del SARS-CoV-2 negativo. El paciente ciertamente tiene carga genética para diabetes tipo 2 y resistencia a la insulina, con genotipos heterocigotos para los SNV en ELMO1 y ATM y VNTR's en $A T X N 2$ y $A T X N 3^{3}$, que conducen a

\section{Correspondencia:}

${ }^{*}$ Carlos E. Cabrera-Pivaral

Sierra Mojada 950

Col. Independencia

Fecha de recepción: 08-01-2021

C.P. 44340 , Guadalajara, Jal., México

E-mail: cabrerapivaral@prodigy.net.mx DOI: $10.24875 / C I R U .21000017$
Cir Cir. 2021;89(4):559-562

Contents available at PubMed www.cirugiaycirujanos.com ajo la licencia CC BY-NC-ND (http://creativecommons.org/licenses/by-nc-nd/4.0/). 
Cirugía y Cirujanos. 2021;89(4)

Tabla 1. Marcadores genéticos y bioquímicos al ingreso del paciente con SARS-CoV-2

\begin{tabular}{|c|c|c|}
\hline Marcadores genéticos & Genotipo & Riesgo \\
\hline Mutación en el factor V Leiden, F5 G1691A & $G / G$ & Normal \\
\hline Polimorfismo de protrombina en 20210, F2 G20210A & $\mathrm{A} / \mathrm{A}$ & Riesgo de hipercoagulabilidad \\
\hline Polimorfismo MTHFR C677T & $\mathrm{C} / \mathrm{T}$ & Normal \\
\hline Polimorfismo MTHFR A1298C & $\mathrm{A} / \mathrm{A}$ & Normal \\
\hline $\begin{array}{l}\text { Polimorfismo Ins/del intrón } 16 \text { del gen para la enzima } \\
\text { convertidora de angiotensina I (ECA-1 l/D) }\end{array}$ & $\mathrm{I} / \mathrm{D}$ & $\begin{array}{l}\text { Riesgo secundario, proinflamatorio vascular, } \\
\text { condicionante de hiperfibrinólisis, aumenta ECA-1, ECA-2 } \\
\text { y la angiotensina II en plasma }\end{array}$ \\
\hline $\begin{array}{l}\text { Polimorfismo (CAG) n en el exón } 1 \text { del gen para la } \\
\text { ataxina } 2 \text { (ATXN2). }\end{array}$ & $22 / 25$ & $\begin{array}{l}\text { Los alelos largos }(>22) \text { o cortos }(<22) \text { están ligados a } \\
\text { resistencia a la insulina, diabetes tipo } 2 \text {, incremento del } \\
\text { número de eritrocitos y del volumen corpuscular medio. } \\
\text { así como hipercoagulabilidad }\end{array}$ \\
\hline $\begin{array}{l}\text { Polimorfismo (CAG) } n \text { en el exón } 10 \text { del gen para la } \\
\text { ataxina } 3 \text { (ATXN3) }\end{array}$ & $25 / 30$ & $\begin{array}{l}\text { La ataxina } 3 \text { participa en la respuesta ante infecciones } \\
\text { virales } \\
\text { Los alelos largos (12-44) se asocian a hiperinsulinemia, } \\
\text { diabetes tipo } 2 \text { y valores elevados de factor de } \\
\text { crecimiento insulínico tipo } 1\end{array}$ \\
\hline Polimorfismo SNV rs11212617 del gen ATM, T>G & $\mathrm{T} / \mathrm{G}$ & $\begin{array}{l}\text { El estado heterocigoto se asocia con resistencia a la } \\
\text { insulina, desarrollo de diabetes tipo } 2 \text { e inadecuada } \\
\text { reparación vascular } \\
\text { El estado homocigoto T se vincula a la respuesta al } \\
\text { tratamiento con metformina }\end{array}$ \\
\hline Polimorfismo SNV rs1345365 del gen ELMO1, G>A & $\mathrm{G} / \mathrm{A}$ & $\begin{array}{l}\text { El estado heterocigoto se asocia con rasgos del síndrome } \\
\text { metabólico y es proinflamatorio vascular } \\
\text { El estado homocigoto AA es protector contra el desarrollo } \\
\text { de diabetes tipo } 2\end{array}$ \\
\hline Estudios de laboratorio y bioquímicos & Nivel & Interpretación \\
\hline Proteína C & $109.42 \%$ & Normal (testigo 102.4) \\
\hline Antitrombina III & $96.12 \%$ & Normal (testigo 94.56) \\
\hline Factor VII & $144.60 \%$ & Normal (testigo 132.6\%) \\
\hline Factor de Von Willebrand & $189.74 \%$ & Incrementado (testigo 125.6) \\
\hline Tiempo parcial de tromboplastina tisular & $26 / 31.3 \mathrm{~s}$ & Normal, radio 0.89 \\
\hline Tiempo de protrombina & $11.8 / 11.8 \mathrm{~s}$ & Normal, radio 0.96 \\
\hline Dímero D & $1001 \mathrm{mg} / \mathrm{l}$ & Incrementado, aumento de la gravedad \\
\hline Fibrinógeno & $1001 \mathrm{mg} / \mathrm{dl}$ & Incrementado \\
\hline Ferritina & $931 \mathrm{ng} / \mathrm{ml}$ & Incrementada \\
\hline Interleucina 6 & $36 \mathrm{pg} / \mathrm{ml}$ & Normal \\
\hline Colesterol total basal en ayuno de $8 \mathrm{~h}$. & $227 \mathrm{mg} / \mathrm{dl}$ & Incrementados (normal 100-240) \\
\hline Triglicéridos totales en ayuno de $8 \mathrm{~h}$ & $399 \mathrm{mg} / \mathrm{dl}$ & Incrementados (normal 35-160) \\
\hline Glucosa sérica basal en ayuno de $8 \mathrm{~h}$ & $270 \mathrm{mg} / \mathrm{dl}$ & Incrementada (normal<100 mg/dl) \\
\hline Hemoglobina glucosilada & $10.5 \%$ & $\begin{array}{l}\text { Buen control metabólico } 5.5-6-8 \% \text {, medio } 6.8-7.1 \% \text { y } \\
\text { pobre }>7.6 \%\end{array}$ \\
\hline Aspartato aminotransferasa & $72 \mathrm{UI} / \mathrm{I}$ & Incrementada \\
\hline Alanina aminotransferasa & $98 \mathrm{UI} / \mathrm{I}$ & Incrementada \\
\hline Lactato deshidrogenasa & $121 \mathrm{UI} / \mathrm{l}$ & Normal \\
\hline
\end{tabular}


Tabla 1. Marcadores genéticos y bioquímicos al ingreso del paciente con SARS-CoV-2 (Continuación)

\begin{tabular}{|c|c|c|}
\hline Marcadores genéticos & Genotipo & Riesgo \\
\hline Lactato sérico & $1.28 \mathrm{mg} / \mathrm{dl}$ & Normal \\
\hline Bilirrubina total & $0.8 \mathrm{mg} / \mathrm{dl}$ & Normal \\
\hline Bilirrubina indirecta & $0.2 \mathrm{mg} / \mathrm{dl}$ & Normal \\
\hline Bilirrubina directa & $0.6 \mathrm{mg} / \mathrm{dl}$ & Normal \\
\hline \multicolumn{3}{|l|}{ Biometría hemática: } \\
\hline Leucocitos & 6.68 millones $/ \mathrm{mm}^{3}$ & Normal \\
\hline Eritrocitos & 4.85 miles/ml & Normal \\
\hline Linfocitos & $8.7 \%$ & Ligeramente aumentados \\
\hline Hemoglobina & $13.4 \mathrm{mg} / \mathrm{dl}$ & Normal \\
\hline Hematocrito & $42.1 \%$ & Normal \\
\hline Plaquetas & 383,000 miles $/ \mathrm{ml}$ & Normal \\
\hline \multicolumn{3}{|l|}{ Gasometría: } \\
\hline $\mathrm{pH}$ & 7.47 & Alcalosis respiratoria compensada \\
\hline $\mathrm{pCO}_{2}$ & $28.1 \mathrm{mmol} / \mathrm{l}$ & \\
\hline $\mathrm{pO}_{2}^{2}$ & 60.9 & \\
\hline $\begin{array}{l}\mathrm{HCO}_{3} \\
\text { Déficit de base }\end{array}$ & $20 \mathrm{mEq} / \mathrm{l}-3$ & \\
\hline Déficit de base & & \\
\hline \multicolumn{3}{|l|}{ Azoados séricos: } \\
\hline Urea & $28.1 \mathrm{mg} / \mathrm{dl}$ & Normales \\
\hline Nitrógeno ureico & 13 mg/dl & \\
\hline Creatinina & $0.8 \mathrm{mg} / \mathrm{dl}$ & \\
\hline \multicolumn{3}{|l|}{ Electrolitos séricos: } \\
\hline Sodio & $136 \mathrm{mEq} / \mathrm{l}$ & Normales \\
\hline Potasio & 4.7 mEq/l & \\
\hline
\end{tabular}

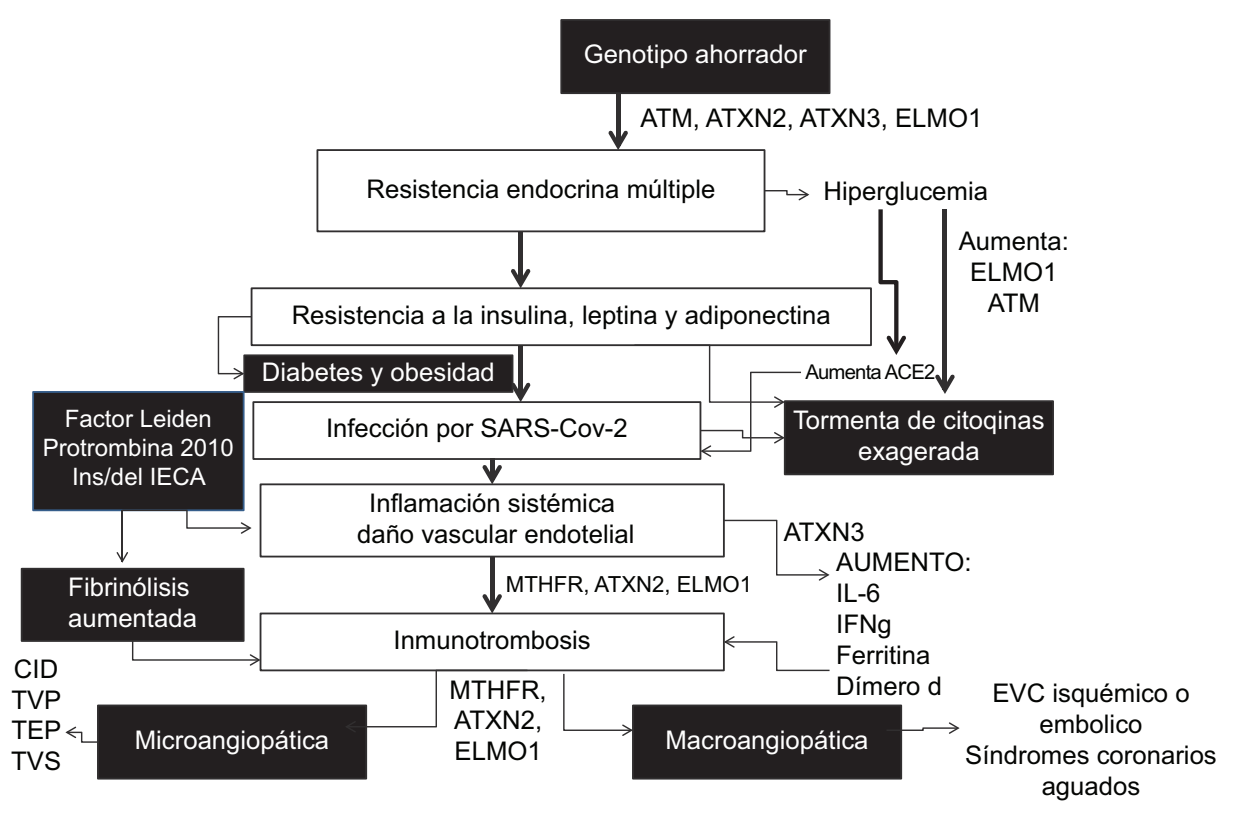

Figura 1. Participación de los marcadores genéticos analizados en la coagulopatía por SARS-CoV-2 en el paciente con diabetes tipo 2. ATM: gen de la ataxia telangiectasia; ATXN2: gen de la ataxina 2; ATXN3: gen de la ataxina 3; CID: coagulación intravascular diseminada; ELMO1: gen dedicador de la citocinesis y alargamiento celular tipo 1; EVC: enfermedad vascular cerebral; IFN- $\gamma$ : interferón gamma; IL: interleucina; MTHFR: gen de la metilentetrahidrofolato reductasa; TEP: tromboembolia pulmonar; TVP: trombosis venosa profunda; TVS: trombosis venosa superficial. 
coagulopatía, estado proinflamatorio vascular, hiperfibrinólisis (Fig. 1) ${ }^{4}$, potenciada por el genotipo I/D de la enzima convertidora de angiotensina y protrombina 20210 homocigoto A, obesidad, dislipidemia, valores elevados del factor de Von Willebrand y fibrinógeno, que explican en conjunto la aparición de la trombosis superficial de la safena, no descrito en pacientes con SARS-CoV-2. Consideramos que, además del dímero D, la interleucina 6 y la ferritina (por debajo del punto de corte en el paciente) que reportan Carrillo-Esper $\mathrm{R}$, et al ${ }^{1}$ como biomarcadores de la coagulopatía, son complementarios los marcadores genéticos de trombofilia y de inflamación analizados, así como las variantes en los genes CBS, VKORC1 y SERPING1, ya que por docking tienen alta afinidad por ORF7a del SARSCoV-2 ${ }^{4,5}$. La respuesta al tratamiento del paciente radicó en el manejo inicial del control glucémico y del hipotiroidismo, y en que es portador de un genotipo viral con menor afinidad por la hemoglobina.

\section{Agradecimientos}

Los autores agradecen a la Fundación Mexicana de Enfermedades Genéticas y Medicina Genómica, AC y al Laboratorio Estatal de Salud Pública, Servicios de Salud, Oaxaca, por el financiamiento de los estudios del paciente.

\section{Responsabilidades éticas}

Protección de personas y animales. Los autores declaran que para esta investigación no se han realizado experimentos en seres humanos ni en animales.

Confidencialidad de los datos. Los autores declaran que han seguido los protocolos de su centro de trabajo sobre la publicación de datos de pacientes.

Derecho a la privacidad y consentimiento informado. Los autores han obtenido el consentimiento informado de los pacientes y/o sujetos referidos en el artículo. Este documento obra en poder del autor de correspondencia.

\section{Financiamiento}

Fundación Mexicana de Enfermedades Genéticas y Medicina Genómica, AC, y Laboratorio Estatal de Salud Pública, Servicios de Salud, Oaxaca.

\section{Conflicto de intereses}

Los autores declaran no tener conflictos de intereses.

\section{Bibliografía}

1. Carrillo-Esper R, Melgar-Bieberach RE, Jacinto SA, Tapia M, Campa AN Alteraciones de la coagulación en COVID-19. Cir Cir. 2020;88:787-93.

2. Godínez SA, Valerdi L. Obesidad: resistencia endocrina múltiple. Revista de Endocrinología y Nutrición. 2012;20:152-68.

3. Feng Q, Miao Y, Ge J, Yuan Y, Zuo Y, Qian L, et al. ATXN3 positively regulates type I IFN antiviral response by deubiquitinating and stabilizing HDAC3. J Immunol. 2018;201:675-87.

4. Flores LJ, Ramirez-Garcia SA, Candelario G. Acute pyelonephritis: the clot unfolds, evidence than supports complex nature of the renal vein thrombosis; therapeutical implications. Brain Disord Ther. 2015;4:168.

5. Holcomb D, Alexaki A, Hernandez N, Laurie K, Kames J, Hamasaki-Katagiri $\mathrm{N}$, et al. Potential impact on coagulopathy of gene variants of coagulation related proteins that interact with SARS-CoV-2. bioRxiv. 2020;2020.09.08.272328. 